\title{
GENEALOGICAL TREES FROM GENETIC DISTANCES
}

\author{
Luce Prignano \\ Dipartimento di Fisica, Università di Roma "La Sapienza", I-00185 Roma, Italy \\ Maurizio Serva \\ Dipartimento di Matematica, Università dell'Aquila, I-67010 L'Aquila, Italy
}

(Dated: November 5, 2018)

\begin{abstract}
In a population with haploid reproduction any individual has a single parent in the previous generation. If all genealogical distances among pairs of individuals (generations from the closest common ancestor) are known it is possible to exactly reconstruct their genealogical tree. Unfortunately, in most cases, genealogical distances are unknown and only genetic distances are available. The genetic distance between two individuals is measurable from differences in mtDNA (mitochondrial DNA) in the case of humans or other complex organisms while an analogous distance can be also given for languages where it is measured from lexical differences. Assuming a constant rate of mutation, these genetic distances are random and proportional only on average to genealogical ones. The reconstruction of the genealogical tree from the available genetic distances is forceful imprecise. In this paper we try to quantify the error one may commit in the reconstruction of the tree for different degrees of randomness. The errors may concern both topology of the tree (the branching hierarchy) and, in case of correct topology, the proportions of the tree (length of various branches).

Pacs:

05.40.-a -Fluctuation phenomena, random processes, noise, and Brownian motion, 87.23.Ge -Dynamics of social systems, 87.23.Kg -Dynamics of evolution, 89.75.Hc -Networks and genealogical trees.
\end{abstract}




\section{INTRODUCTION}

Haploid reproduction implies that any individual has a single parent in the previous generation. Since some of the individuals may have the same parent, the number of ancestors of the present population decreases going backwards in time until a complete coalescence to a single ancestor [7, 8, 18]. Therefore, it is possible to construct a genealogical tree whose various branching events connect all the individuals living in the present time to the single founder ancestor. The genealogical distance between two individuals is simply the time from their last common ancestor and it may assume the maximal value only when the common ancestor coincide with the founder.

In the limit of infinite population size, most of the quantities remain random, for example, this is the case of the probability density of genealogical distances in a single population. In fact, even in the thermodynamic limit, this quantity varies for different populations or, at different times, for the same population. The discovery of this non self-averaging behavior is due to the pioneering work of Derrida, Bessis, Jung-Muller and Peliti [2, 3, 4].

Nevertheless, if all genealogical distances among pairs of individuals are known it is possible to exactly reconstruct their genealogical tree. Unfortunately, in practice, genealogical distances are unknown unless one has the relatives historical records which is not the case of living organisms populations and of most of the linguistic groups (Latin languages are an exception). In most cases, only genetic distances are available. These distances, in the case of humans or other complex organisms, can be measured from the difference in mtDNA (mitochondrial DNA), which is inherited only from the mother and, therefore, it undergoes to haploid reproduction [9, 10]. In the case of languages, instead, they are deduced from lexical differences [12, 13, 14]. Assuming a constant rate of mutation, these genetic distances are random and they are proportional only on average to genealogical distances. The reconstruction of the genealogical tree from the available genetic distances is forceful imprecise. In this paper we try to quantify the error one may commit in the reconstruction of the tree for different degrees of randomness. The errors may concern both topology of the tree (the branching hierarchy) and, in case of correct topology, the proportions of the tree (length of various branches). The paper is organized as follows: sections 2 is devoted to the deterministic process which is associated to the genealogical distances. We also show there how to exactly reconstruct the genealogical tree form them. In section 3 we define 
and discuss the random process associated to the genetic distances. In section 4 we introduce a measure of topological distance between two tree and we quantify how topologically wrong is the tree reconstructed from genetic distances. In sections 5 we quantify the error concerning the length of various branches of topologically correct trees. Finally section 6 contains conclusions and outlook. The paper is completed by an appendix where we have moved some lengthy calculations.

\section{DYNAMICS OF GENEALOGICAL DISTANCES AND TREES RECONSTRUCTION}

We consider a very general model of a population of constant size $N$ whose generations are not overlapping in time: any generation is replaced by a new one and any individual has a single parent. The stochastic rule which assigns the number of offspring to any individual can be chosen in many ways. In fact, for large population size, results do not depend on the details of this rule, the only requirement is that the probability of having the same parent for two individuals must be of order $1 / N$ for large $N$. Here we choose the Wright-Fisher rule: any individual in the new generation chooses one parent at random in the previous one, independently on the choice of the others.

The genealogical distance between two given individuals is the number of generations from the closest common ancestor. For large $N$ distances are proportional to $N$, it is then useful to re-scale them dividing by $N$.

So let us define $d(\alpha, \beta)$ as the rescaled genealogical distance between individuals $\alpha$ and $\beta$ in a population of size $N$. For two distinct individuals $\alpha$ and $\beta$ in the same generation one has

$$
d(\alpha, \beta)=d(g(\alpha), g(\beta))+\frac{1}{N},
$$

where $g(\alpha)$ and $g(\beta)$ are the parents of $\alpha$ and $\beta$ respectively. Accordingly with the WrightFisher rule, parents are chosen among all possible ones with equal probability $1 / N$ and, therefore, $g(\alpha)$ and $g(\beta)$ coincide with probability $1 / N$. In this case the distance $d(g(\alpha), g(\beta))$ vanishes. On the contrary, the parents of $\alpha$ and $\beta$ are distinct individuals $\alpha^{\prime}$ and $\beta^{\prime}$ with probability $(N-1) / N$. The above equation, when considered all the $N(N-1) / 2$ pairs, entirely defines the dynamics of the population and simply states that the rescaled distance in the new generation increases by $1 / N$ with respect to the parents distance. Briefly, $d(\alpha, \beta)=1 / N$ 
with probability $1 / N$ and $d(\alpha, \beta)=d\left(\alpha^{\prime}, \beta^{\prime}\right)+1 / N$ with probability $(N-1) / N$.

This equation can be iterated for any of the possible $N(N-1) / 2$ initial pairs $\alpha$ and $\beta$, which correspond to the entries of an upper triangular matrix. The iteration stops when there is a coincidence of parents and in this way all the distances $d(\alpha, \beta)$ can be calculated.

Shortly: iteration of equation 1 gives as output the realization of the random $N(N-1) / 2$ distances $d(\alpha, \beta)$ which are the entries of an upper triangular matrix containing all the necessary information for the reconstruction of the genealogical tree of the population. The tree is completely identified by its topology and by the time separation of all branching events. There exist many methods that can be used for this reconstruction, a simple one is the Unweighted Pair Group Method Average (UPGMA) [17]. This algorithm works as follows: it first identifies the two individuals with shortest distance, and put their branching at their time separation. Then, it treats this pair as a new single object whose distance from the other individuals is the average of the distance of its two components. Subsequently, among the new group of objects it identifies the pair with the shortest distance, and so on. At the end, one is left with only two objects which represents the two main branches, whose distance gives the time position of the root of the tree. Then, the time from the last common ancestor of all individuals in the populations results fixed.

This method works for any kind of upper triangular matrix representing distances among pairs of individuals, not necessarily originated by the coalescent process. In the coalescent case, nevertheless, the method gives the correct tree reproducing the historical branching events and the correct time separations among them. Notice, that at any time it chooses two individuals with shortest distance. Then, it is easy to realize that for the coalescent the distance of the two individuals from any third one is the same. Therefore, in this case, all UPGMA averages are between pairs with identical distances so that also the resulting new common distances are the same.

Genealogical trees are very complex objects and genealogical distances are distributed according to a probability density which remains random in the limit of large population [15, 16]. Anyway, the mathematical theory of coalescent gives us the ability to deduce some important information about their statistical structure. Consider a sample of $n$ individuals in a population of size $N$, where $N$ is very large with respect to $n$. The probability that they all have different parents in the previous generation is $\prod_{k=0}^{n-1}\left(1-\frac{k}{N}\right)$. Therefore, the probability that their ancestors are still all different in a past time $t$ corresponding to $t N$ generations is 
$\left[\prod_{k=0}^{n-1}\left(1-\frac{k}{N}\right)\right]^{t N}$. If $N$ is large compared to $n$ this quantity is approximately $\exp \left(-c_{n} t\right)$ where

$c_{n}=\frac{n(n-1)}{2}$. The genealogical tree results from this rule: the average probability density for the time lag for the coalescent event for $n$ individuals is $p_{n}(t)=c_{n} \exp \left(-c_{n} t\right)[1,7]$.

Therefore, the tree starts at the root with the two main branches, then, the following branching event is at a random time lag $t_{2}$ with probability density $p\left(t_{2}\right)=e^{-t_{2}}$ and after this time the tree has three branches (see Fig. 3). The next branching is after a time lag $t_{3}$ with probability density is $p\left(t_{3}\right)=3 e^{-3 t_{3}}$ and then the tree has four branches, and so on.

\section{RANDOM COALESCENT PROCESS}

As already mentioned, in the coalescent model, genealogical distances measure the time from the last common ancestor of two individuals. Nevertheless, in almost all real situations, we have to deal with genetic distances reconstructed from directly measurable empirical quantities.

In case of complex organisms, mtDNA is inherited only from the mother and, therefore, it undergoes to haploid reproduction, so in this case genetic distances are proportional to the number of mutations occurred in the compared mtDNA sequences (see, for example, [9, 10]). Analogously languages can be considered as haploid individuals whose vocabulary changes accumulate in time, in this case genetic distances can be evaluated by lexical distances $\lfloor 12$, 13, 14].

In both cases, an individual randomly accumulates mutations at a constant rate and the genetic distance of a pair of individuals is the sum of the mutations that they accumulated since their last common ancestor (rescaled by $N$ ). As a consequence, genetic distances are proportional only on average to genealogical ones. Therefore, we have to modify the deterministic equation (11) in order to take into account this randomness. We may assume that increments in the genetic distance have the simple form

$$
h(\alpha, \beta)=h(g(\alpha), g(\beta))+\gamma_{\alpha}+\gamma_{\beta}
$$

where $g(\alpha)$ and $g(\beta)$ are the parents of $\alpha$ and $\beta$ respectively, while $\gamma_{\alpha}$ and $\gamma_{\beta}$ are random variables associated to the mutations of $\alpha$ and $\beta$. They are zero if the genome of the parent is transmitted unaltered and a positive constant if a mutation occurs. We assume that the probability is $1-\frac{\mu}{2 N}$ for zero and $\frac{\mu}{2 N}$ for the positive constant $\frac{1}{\mu}$. In a compact form: 


$$
\gamma_{\alpha}=\left\{\begin{array}{c}
0 \quad \text { prob }=1-\frac{\mu}{2 N} \\
\frac{1}{\mu} \quad \text { prob }=\frac{\mu}{2 N}
\end{array}\right.
$$

This rule grants that genetic distances are equal to genealogical distances on average, in fact, the expected value of the sum $\gamma_{\alpha}+\gamma_{\beta}$ is $1 / N$.

Notice that we compare genealogical distances generated by (1) with genetic ones generated by (2). Since they describe two aspects of the same population, the family history must be the same. This means that the realization of the part of the process which assigns parents in (1) and in (2) must also be the same $(\alpha \rightarrow g(\alpha))$, the only difference lays in the deterministic/random nature of the distance increment. In other words, the parents of two individuals $\alpha$ and $\beta$ are unequivocally assigned. Then, their genealogical distance is $d(\alpha, \beta)=d(g(\alpha), g(\beta))+1 / N$, while the genetic one is $h(\alpha, \beta)=h(g(\alpha), g(\beta))+\gamma_{\alpha}+\gamma_{\beta}$ where $\gamma_{\alpha}$ and $\gamma_{\beta}$ are the previously defined random variables.

In order to have a qualitative idea of the differences between the set of genetic distances and the set of genealogical ones we plot in Fig.1 the frequency of the distances of the two sets. We have used the same realization of the process for 700 individuals and we have chosen $\mu=50$ for the genetic distances. We can see that while genealogical distances may assume only few values where their distribution has spikes [15, 16], the genetic ones are dispersed around them. The dispersion decreases when $\mu$ increases and when $\mu=2 N$ genetic distances lose their randomness in mutations, and they equal, for any realization, the associated genealogical ones ( compare (11) with (2) and (3) ). In case of a very large population $(N \rightarrow \infty)$ the coincidence of the two sets of distances is recovered in the $\mu \rightarrow \infty$ limit.

\section{WRONG TREE RECONSTRUCTION: TOPOLOGY}

The problem, in most practical cases, is that genealogical distances are unknown and one would like to reconstruct the genealogical tree of a population from the measured genetic distances. This is the case of biology where strands of mtDNA are compared as well of lexicostatistics where vocabularies or grammar structures take the same role of mtDNA.

As mentioned in previous section, when $\mu=2 N$ equations (11) and (2) coincide and randomness in mutations is lost. In this limiting case genetic and genealogical distances 

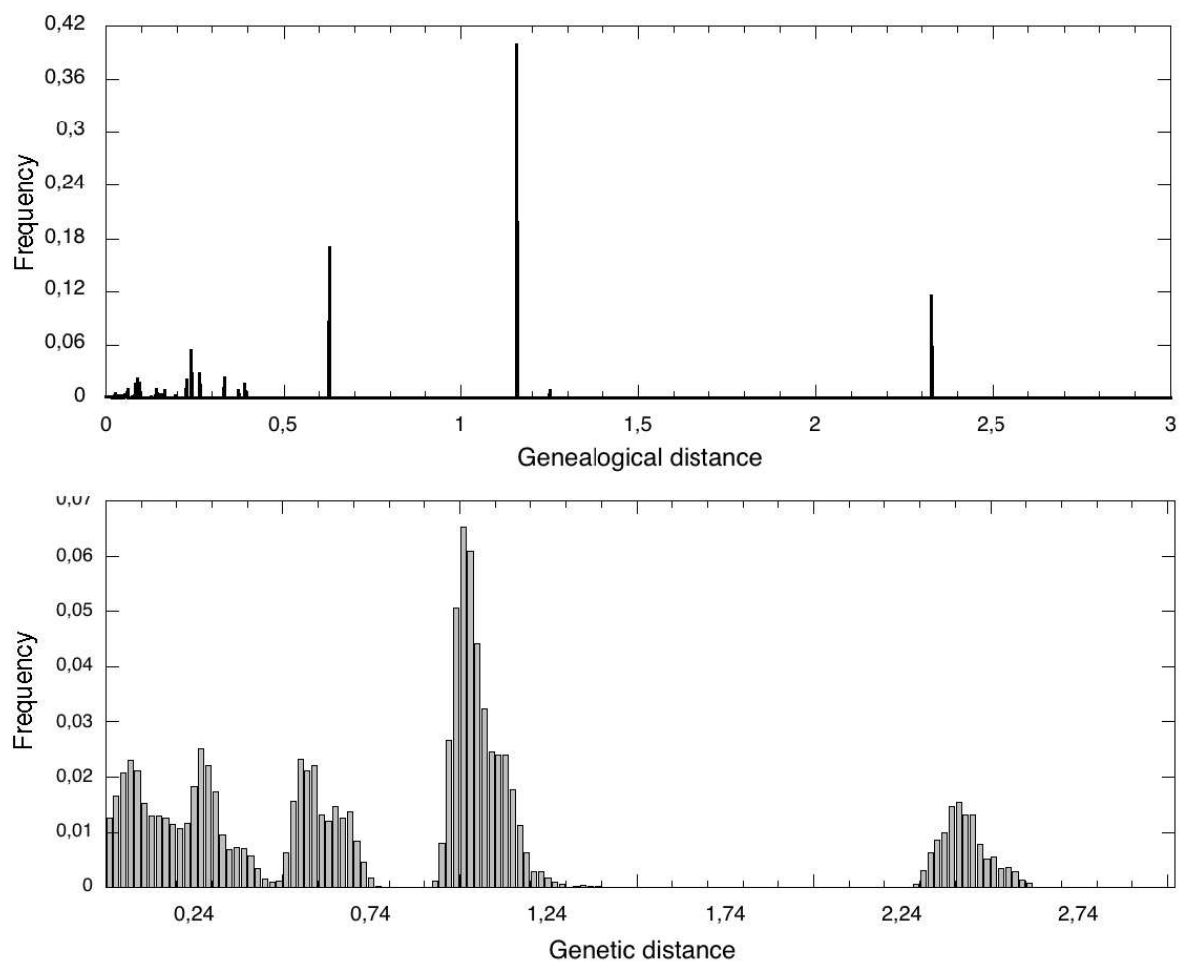

FIG. 1: Frequencies of the genealogical and genetic distances $(\mu=50)$ in a population of 700 individuals. The realization of the genealogical process (the family history) is the same for the two distributions.

are equal and not only the frequency distributions are identical, but also the family trees reconstructed by UPMGA will be exactly the same. For smaller values of $\mu$, we expect that the fidelity level of reconstruction of a tree decreases. Then, we would like to have a quantitative information on the difference between the trees reconstructed from the matrices of genealogical and genetic distances.

A qualitative understanding of the problem is immediate from Fig. 22 where four trees of twenty leaves are reconstructed. The first and correct one with label $A$, is associated to the genealogical distances and the remaining three to the genetic ones for three different values of $\mu$. The realization of the genealogical process (the family history) is the same for the four pictures. One can see that the quality of the reconstruction decreases for smaller $\mu$. In fact, the tree with label $D$, which corresponds to $\mu=100$, is topologically quite correct, with a couple of wrong clades (see leaves $G, B, T, C, A$ and $M, U, E$ ) at the lower level. Also the separation times of the branches are not so different from the correct ones. For $\mu=50$ with 
label $C$ and $\mu=10$ with label $B$ the quality of reproduction reduces, clades are wrong also at a higher level and times depths are quite different from the right ones.
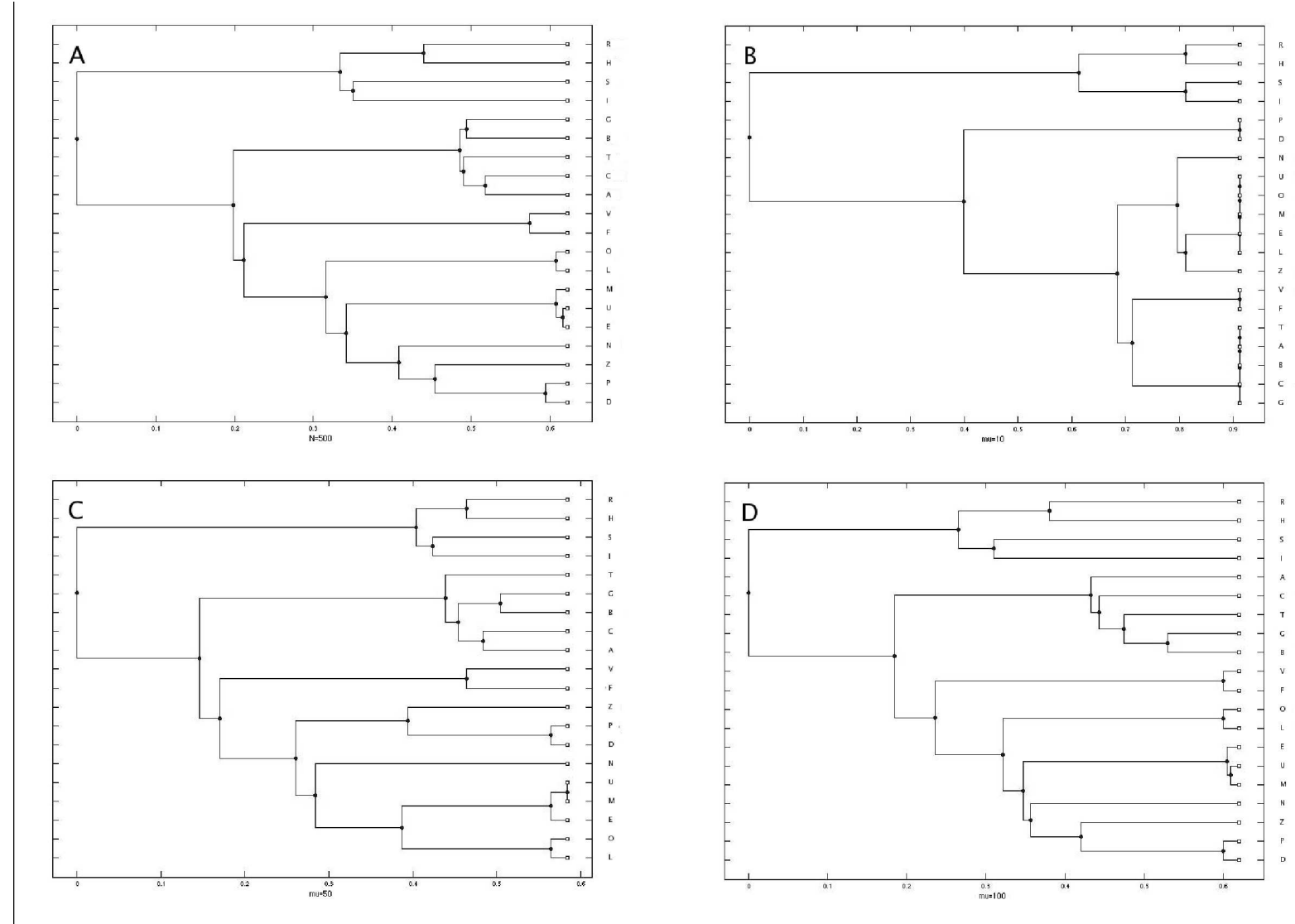

FIG. 2: Four trees of twenty leaves, corresponding to a sample of 20 individuals in a population of 500. The first tree is reconstructed from the genealogical distances and the others from the genetic ones ( $\mu=10$ for $B, \mu=50$ for $C$ and $\mu=100$ for $D$ ). The quality of the reproductions of the first tree (the correct one) by the others is lower for smaller $\mu$ both for what concerns topology and time depths.

We start the quantitative study of the quality of reconstruction considering the simplest situation of tree with three leaves. The topology of a three leaves tree is completely determinate by the pair of individuals that first match together because their distance is the smallest. Consequently, the genealogical and the genetic trees reconstructed by the UPGMA will have the same topology if the same pair of individuals has both the smallest genetic and genealogical distance. Let us call $\alpha, \beta$ and $\gamma$ the three individuals, and assume that $\alpha$ and $\beta$ are the pair with the smallest genealogical distance $d(\alpha, \beta)$. By the argument in 
Section 2 we know that $d(\alpha, \beta)=t_{3}$ and $d(\alpha, \gamma)=d(\beta, \gamma)=t_{2}+t_{3}$, where $t_{2}$ and $t_{3}$ are independent exponentially distributed variables with average 1 and $1 / 3$ respectively. Then let us consider the two following events concerning genetic distances, the first that we call $A$ is

$$
h(\alpha, \beta)<\min \{h(\alpha, \gamma) ; h(\beta, \gamma)\}
$$

If $A$ is satisfied, the topology of the genetic tree reconstructed by UPGMA is the correct one since it is the same of that of the genealogical tree. The second that we call $B$ is

$$
\begin{gathered}
h(\alpha, \beta)=\min \{h(\alpha, \gamma) ; h(\beta, \gamma)\} \\
h(\alpha, \gamma) ; \neq h(\beta, \gamma)
\end{gathered}
$$

which corresponds to an ambiguous (but unlikely) situation for UPGMA which will be able to reconstruct correctly the tree with probability $1 / 2$. The third that we call $C$ will be

$$
h(\alpha, \beta)=h(\alpha, \gamma)=h(\beta, \gamma)
$$

which is also ambiguous (and even more unlikely). In this case, UPGMA will be able to reconstruct correctly the tree with probability $1 / 3$.

Let us now call $P\left(A \mid t_{2}, t_{3}\right)$ the probability of the event $A$ given the realized values $t_{2}$ and $t_{3}$, and $P\left(B \mid t_{2}, t_{3}\right)$ and $P\left(C \mid t_{2}, t_{3}\right)$ the equivalent conditional probabilities for the events $B$ and $C$ respectively. Let us also call $P\left(W \mid t_{2}, t_{3}\right)$ the probability of a wrong reconstruction of the tree correspondingly to $t_{2}$ and $t_{3}$. We have

$$
P\left(W \mid t_{2}, t_{3}\right)=1-P\left(A \mid t_{2}, t_{3}\right)-\frac{1}{2} P\left(B \mid t_{2}, t_{3}\right)-\frac{1}{3} P\left(C \mid t_{2}, t_{3}\right)
$$

Now we call $n(\alpha)$ the number of mutations along the branch $\alpha$ divided by $\mu$, as shown in Fig. 3, analogously we define $n(\beta), n(\gamma)$ and $n(\alpha \beta)$ as the numbers of mutations divided by $\mu$ along the branches indicated in Fig. 3 ,

We will have $h(\alpha, \beta)=n(\alpha)+n(\beta), h(\alpha, \gamma)=n(\alpha)+n(\alpha \beta)+n(\gamma)$ and $h(\beta, \gamma)=$ $n(\beta)+n(\alpha \beta)+n(\gamma)$. The advantage is that the four new variables are independent and can be obtained as the sum of variables of type (3) where the sum goes on a number which is $N$ times the time lag of the associated branch. Namely, $t_{3}$ for $n(\alpha)$ and $n(\beta), t_{2}$ for $n(\alpha \beta)$ and $t_{2}+t_{3}$ for $n(\gamma)$. 


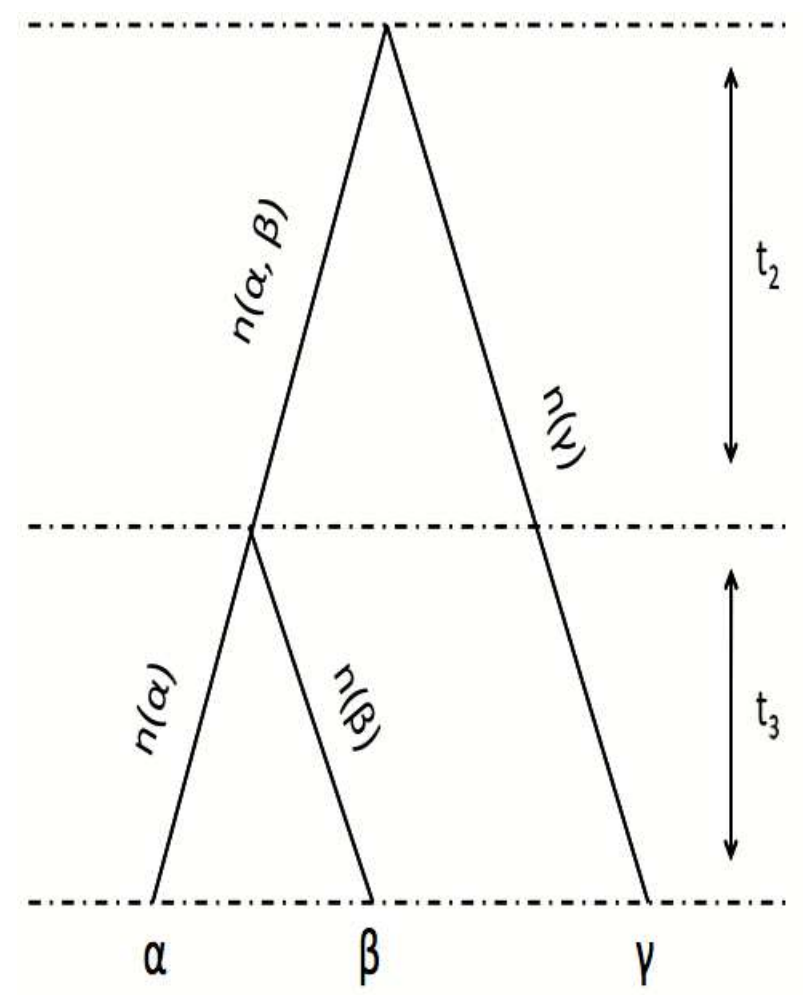

FIG. 3: Outline of a three leaves tree. $n(\alpha), n(\beta), n(\gamma)$ and $n(\alpha \beta)$ are the numbers of mutations divided by $\mu$.

Given this construction we can trivially but painfully compute (see the Appendix) the conditional probability $P\left(W \mid t_{2}, t_{3}\right)$ and, then, the absolute probability of a wrong tree $P(W)$ as the marginal of the joint probability $P\left(W \mid t_{2}, t_{3}\right) p\left(t_{2}\right) p\left(t_{3}\right)$ where $p\left(t_{2}\right)$ and $p\left(t_{3}\right)$ are the exponential densities previously described. The probability of a wrong tree $P(W)$ is plotted in Fig. 4 with respect to the parameter $\mu$ in the case of three individuals in a large population $(N \rightarrow \infty)$.

If we take into account more than three individuals the situation immediately becomes more complicated since the possible tree topologies increase exponentially with the number of leaves. So we need to introduce a measure of difference between the genealogical tree and an associated genetic one. The simplest tree distance measure is the Robinson-Foulds Symmetric Difference [11], which only depends on the topology of the two tree and not on the differences in branches length.

The Symmetric Difference (SD) is computed by considering all possible branches that may exist in the two trees. Each inner branch, i.e. a branch connecting two nodes or one 


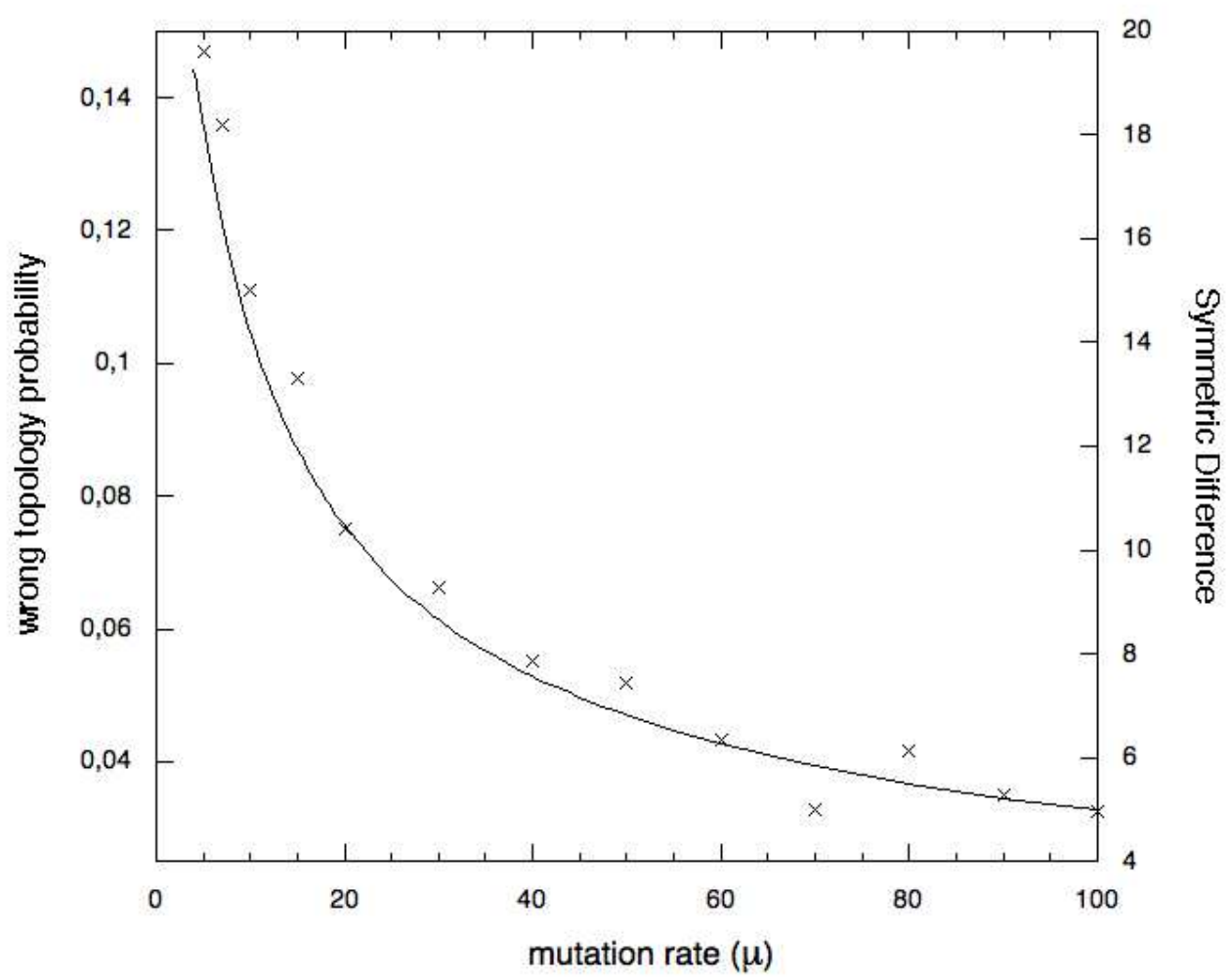

FIG. 4: Expected Symmetric Difference between a genealogical tree and the associated genetic tree plotted with respect $\mu$. The full line corresponds to the probability of a wrong topology $\mathrm{P}(\mathrm{W})$ for a three leaves tree while crosses correspond to the ESD of a 20 leaves tree, estimated numerically. In the first case, computed exactly, the ESD is twice the probability of a wrong topology.

node to the root, identifies a clade in the set of leaves. The resulting distance is simply the number of clades present in one of the considered trees but not in the other. Therefore, two identical trees have zero SD, but it is sufficient to exchange two leaves on one of them to have a non zero SD.

In general SD has not an immediate statistical interpretation, i.e. we cannot say whether a larger distance is significantly larger than a smaller one. Anyway, in the particular case of trees with only three leaves the expected symmetric distance is twice the wrong topology probability $P(W)$. In fact, in a three leaves tree there is only one clade and the Symmetric Distance is equal to 0 in the case of correct topology (if both trees have the same clade) and is equal to 2 in the case of wrong one (if clades are different). Consequently, in this simple case, the expected SD (that we call ESD) is given by the relation 


$$
\mathrm{ESD}=2 \cdot P(W)+0 \cdot(1-P(W))=2 \cdot P(W)
$$

In order to compute numerically the ESD between a genealogical tree and the associated genetic one with parameter $\mu$ we use the following procedure: we take 20 individuals in a population of 500 (a large one) and we use UPGMA to reconstruct their genealogical tree from a realization of the genealogical distances matrix. Then, we construct several associated genetic trees ( 5 for $\mu<15,10$ for greater values) and we compute their averaged $\mathrm{SD}$ with respect to the associated genealogical tree. We start again with a new realization of the matrix of genealogical distances and we repeat the procedure, ending with a new averaged SD. We do it many times (from 6 for $\mu=5$ to 30 for $\mu=100$ ) and, finally, we take the mean of all averaged SD and we end with a quantity that should be very close to ESD. The number of genealogical trees and that of the associated genetic trees that we use for estimating the ESD increases with $\mu$ since we observe an increasing fluctuation in the $\mathrm{SD}$ values.

In Fig. 4 we plot the estimated ESD of the 20 leaves tree. We also plot the exactly computed $P(W)$ of a three leaves tree which is one half its ESD. We find out, unexpectedly, that they only differ for a factor due to the total number of clades, which depends on the number of leaves.

\section{WRONG TREE RECONSTRUCTION: BRANCHES LENGTH}

Now we study the problem of differences among branches length in genealogical and genetic trees. We first derive the probability of the error between the genetic and genealogical distances of two individuals, and then we will sketch out the computation of the errors for the distances in a tree of three individuals. We will consider only the cases in which genetic and genealogical tree have the same topology. In this way the integral of the probability of the error (errors for the three leaves case) will be equal to the total probability of having the right topology $P(R)=1-P(W)$. Obviously, the right topology condition is always satisfied for two individuals $(P(R)=1)$.

In a tree of two individuals we have only a single genealogical distance to be compared with a single genetic one. Let us call $t$ the genealogical distance and $h$ the genetic one, that is the number of mutations on both the genealogical branches divided by $\mu$. We call $k=\mu h$ 
the total number of mutations on these two branches, then $t$ and $h$ will be equal only if $k=\mu t$. By using equations (3) we can write the expression of the conditional probability of having $k$ mutations along the two genealogical branches with lengths $t N$ and, therefore, total length $2 t N$ :

$$
p_{\mu}(k \mid 2 t N)=\left(\begin{array}{c}
2 t N \\
k
\end{array}\right)\left(\frac{\mu}{2 N}\right)^{k}\left(1-\frac{\mu}{2 N}\right)^{2 t N-k} \sim \frac{e^{-\mu t}(\mu t)^{k}}{k !} .
$$

where the approximation holds for large $N$.

One easily gets the conditional averages $\langle k\rangle=\mu t$ and $\left\langle(k-<k>)^{2}\right\rangle=\mu t$. It is than straightforward to define the error between genetic and genealogical distance as $\epsilon=\frac{h-t}{\sqrt{t}}$, in this way, in fact, one gets the conditional (with respect to t) averages $\langle\epsilon\rangle=0$ and $<\epsilon^{2}>=1 / \mu$ which do not depend on $t$. As a consequence of this independence the absolute averages of $\epsilon$ and $\epsilon^{2}$ coincide with the conditional ones. The conclusion is that the typical error in evaluating the distance from the common ancestor grows linearly with $1 / \sqrt{\mu}$

The independence of the two conditional averages from $t$ does not implies that the conditional probability density for $\epsilon$ is itself independent on $t$. In fact, since one has $h=k / \mu$ and, therefore, $\epsilon=\frac{k-\mu t}{\mu \sqrt{t}}$, the conditional probability density of the error turns out to be

$$
p_{\mu}(\epsilon \mid t)=\sum_{k=0}^{\infty} \frac{e^{-\mu t}(\mu t)^{k}}{k !} \delta\left(\epsilon-\frac{k-\mu t}{\mu \sqrt{t}}\right) .
$$

where the $\delta(\cdot)$ are Dirac delta functions. This conditional density for $\epsilon$ given a genealogical distance $t$, at variance with its two first moments, clearly depends on $t$.

Finally, the absolute probability density $p_{\mu}(\epsilon)$ can be calculated as the marginal of the joint probability density $p_{\mu}(\epsilon \mid t) p(t)$, where $p(t)=\exp (-t)$ is the density of the genealogical distances. We obtain

$$
p_{\mu}(\epsilon)=\sum_{k=0}^{\infty} \frac{e^{-(\mu+1) l}(\mu l)^{k}}{k !} \frac{2 \mu l \sqrt{l}}{\mu l+k} .
$$

where we have to use for $l$ the following definition

$$
\sqrt{l}=\frac{\sqrt{(\epsilon \mu)^{2}+4 \mu k}-\epsilon \mu}{2 \mu} .
$$

In Fig. $5 p_{\mu}(\epsilon)$ is plotted for some values of $\mu$. In the limit $\mu \rightarrow \infty$ the distribution becomes a Dirac delta function centered in zero according with the fact that the variance goes to zero as $1 / \sqrt{\mu}$. 


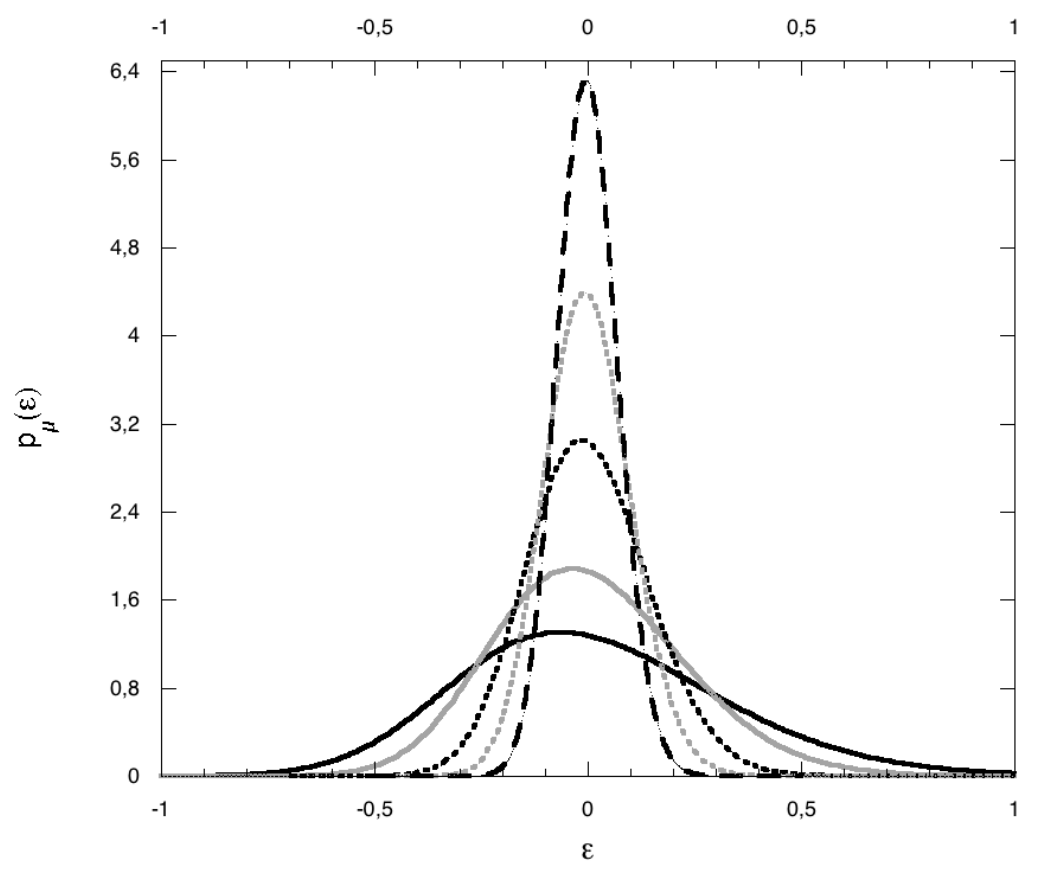

FIG. 5: The probability distribution of the error $p_{\mu}(\epsilon)$ is plotted for $\mu=10, \mu=20, \mu=50$, $\mu=100$ and $\mu=200$. The curves become sharper for increasing values of $\mu$.

Now, let us come back to the case of a genealogical tree with three leaves. The study of this kind of structure, even if much simpler than the genealogical tree of an entire population, gives us important information since concerns the reconstruction of top of the tree. Indeed, to reconstruct in the correct way this part of the genealogical tree, that is the part going from the founder to the three most recent ancestors of the entire population, means to rightly identify the three main sub-populations and their separation times.

We have seen the probability of having the correct topology for a three leaves tree from the genetic matrix by using UPGMA. In the following, we will give a sketch of the derivation of the errors distribution of its two characteristic distances. Hereafter we restrict our analysis to the non-ambiguous situation in which the topology of the genetic tree is the same as that of the genealogical one. We will refer to the scheme in Fig. 3 and to the notation used in Section 4 .

The genetic tree can be characterized by two distances: $h=h(\alpha, \beta)$, separating the 
individuals with minimal genealogical distance $t_{3}$ and $H=[h(\alpha, \gamma)+h(\beta, \gamma)] / 2$, calculated by UPGMA as the mean value of the major distances and therefore separating the third individual from the others. The distance $H$ does not correspond to the maximum genetic distance but it is the mean value of the two major distances and gives a better estimate of the coalescence time $T=t_{3}+t_{2}$ of three individuals to the common ancestor.

We have seen that on average $h$ coincides with $t_{3}, H$ on average is equal to $T$, but, in general, the genetic distances will be different from the genealogical ones and the branches of the tree reconstructed from the genetic matrix by UPGMA will not have the same length as those of the genealogical tree.

Here we define, in analogy with the two leaves case, the errors $\epsilon_{1}$ and $\epsilon_{2}$ of $h$ and $H$ by the equations:

$$
\begin{aligned}
\epsilon_{1} & =\frac{h-t_{3}}{\sqrt{t_{3}}} ; \\
\epsilon_{2} & =\frac{H-T}{\sqrt{T}} .
\end{aligned}
$$

The variables $\epsilon_{1}$ and $\epsilon_{2}$ vanish when the genetic distances equal the genealogical ones.

In order to compute the probability density of the errors we use the relations introduced in previous section and we rewrite the relations (12) in the form

$$
\begin{gathered}
\epsilon_{1}=\frac{n(\alpha)+n(\beta)-t_{3}}{\sqrt{t_{3}}} ; \\
\epsilon_{2}=\frac{n(\alpha)+n(\beta)+2 n(\alpha \beta)+2 n(\gamma)-2 T}{2 \sqrt{T}} .
\end{gathered}
$$

Since $n(\alpha), n(\beta)$ and $n(\gamma)+n(\alpha \beta)$ are independent variables which we described in previous section it is straightforward but painful to compute the conditional probability $p_{\mu}=p_{\mu}\left(\epsilon_{1}, \epsilon_{2} \mid t_{2}, t_{3}\right)$.

The sketch goes as follows: first we write the joint conditional probability for the independent variables $n(\alpha), n(\beta)$ and $n(\gamma)+n(\alpha \beta)$ as

$$
p_{\mu}\left(n(\alpha) \mid t_{3}\right) p_{\mu}\left(n(\beta) \mid t_{3}\right) p_{\mu}\left(n(\gamma)+n(\alpha \beta) \mid-2 t_{2}-t_{3}\right)
$$

where the explicit expression for the probabilities $p_{\mu}(n \mid t)$ is shown in the Appendix (see $(22))$.

Then we are able to compute $p_{\mu}=p_{\mu}\left(\epsilon_{1}, \epsilon_{2} \mid t_{2}, t_{3}\right)$ as the sum $p_{\mu}=p_{\mu}(A)+\frac{1}{2} p_{\mu}(B)+\frac{1}{3} p_{\mu}(C)$ where $p_{\mu}(A)$ is obtained by the sum of the conditional probability (14) over all the triplets $n(\alpha), n(\beta)$ and $n(\gamma)+n(\alpha \beta)$ which satisfy condition $\mathrm{A}$ of section 4 and relations (13). 
Analogously, we can compute $p_{\mu}(B)$ and $p_{\mu}(C)$. Once we have $p_{\mu}\left(\epsilon_{1}, \epsilon_{2} \mid t_{2}, t_{3}\right)$ we can compute the joint probability

$$
p_{\mu}\left(\epsilon_{1}, \epsilon_{2}, t_{2}, t_{3}\right)=p_{\mu}\left(\epsilon_{1}, \epsilon_{2} \mid t_{2}, t_{3}\right) 3 e^{t_{2}+3 t_{3}}
$$

and after integration over $t_{2}$ and $t_{3}$ we obtain the joint density $p_{\mu}\left(\epsilon_{1}, \epsilon_{2}\right)$. The normalization of this density equals the probability $P(R)$ of correct topology identification by UPGMA.

More complex tree could be considered in principle, both for what concerns topology and branches length, nevertheless, the number of calculations increases exponentially.

\section{CONCLUSIONS AND OUTLOOK}

The inner randomness of genetic mutations is an obstacle for a safe reconstruction of a genealogical tree. A wrong reconstruction is more probable the smaller is the probability of a mutation. This is a serious problem since in many cases in biology the distances are measured by a molecular clock which is obtained comparing short strands of DNA which slowly accumulate errors along reproduction events (see, for example, [9, 10]).

We are able to quantify, in simple but relevant cases, the probability of a wrong reconstruction of a tree, both for what concerns the topology and the proportions. We can, for example, give the error concerning the time separation of two species using results in section 5 and we are also able to decide the probability of a wrong reconstruction for a family tree of three species using results in section 4 .

We plan to continue this investigation in order to better quantify the difference between the genetic and the genealogical matrices of distances. We think for example to the possibility of introducing a measure of distance between the associated probabilities of distances. Even more important, it is to find a method to estimate the value of the parameter $\mu$ given an empirical distribution of genetic distances. This would be useful for situations in which the value of $\mu$ is not known a priori as, for example, for the languages in the Indo-European and Austronesian groups [12, 13, 14]. This method would allow us to use the results of this paper for evaluating the fidelity level of phylogenetic trees reconstructed from empirical lexical distances. 


\section{ACKNOWLEDGMENTS}

We thank Filippo Petroni for many discussion concerning both the ideas contained in this paper and their numerical implementation.

\section{APPENDIX: WRONG TOPOLOGY PROBABILITY P(W)}

In this appendix we will use the notation introduced in Section 3 and shown in Fig. 3 , We have that the probability $P(R)$ of having the correct genealogical tree from the genetic distances matrix is:

$$
P(R)=P(A)+\frac{1}{2} P(B)+\frac{1}{3} P(C) .
$$

$P(A)$ is the probability of event $A$, i.e., the probability of having genetic distances among individuals $\alpha, \beta$ and $\gamma$ satisfying the inequality (44). Using the independent variables (4) rewrites as

$$
\max \{n(\alpha) ; n(\beta)\}<n(\alpha \beta)+n(\gamma)
$$

$P(B)$ is the probability of event $B$, while $P(C)$ is the one of event $C$. The events $B$ and $C$ occur respectively if the genetic distances satisfy the conditions (5) and (6) which rewrite respectively as

$$
\begin{gathered}
\max \{n(\alpha) ; n(\beta)\}=n(\alpha \beta)+n(\gamma) ; \\
n(\alpha) \neq n(\beta) .
\end{gathered}
$$

and

$$
n(\alpha)=n(\beta)=n(\alpha \beta)+n(\gamma)
$$

Let us define $n_{1}$ the maximum between $n(\alpha)$ and $n(\beta), n_{2}$ the minimum $\left(n_{1} \geq n_{2}\right)$, and $n_{3}=n(\alpha \beta)+n(\gamma)$. Then, the probability $P(A)$ corresponds to the probability of having $n_{1} \geq n_{2}$ and $n_{1}<n_{3}$. Therefore, we have to determine the total probability for the set of triplets $\left\{n_{1} ; n_{2} ; n_{3}\right\}$, where the variables $n_{i}$ can take only values multiple of $1 / \mu$ and have to satisfy the conditions $n_{1} \in[0, \infty), n_{2} \in\left[0, n_{1}\right]$ and $n_{3} \in\left(n_{1}, \infty\right)$.

If we consider separately the cases $n_{1}<n_{2}$ and $n_{1}=n_{2}$ we immediately obtain:

$$
P(A)=2 \sum_{n_{1}=1 / \mu}^{\infty} \sum_{n_{2}=0}^{n_{1}-1 / \mu} \sum_{n_{3}=n_{1}+1}^{\infty} p_{\mu}\left(n_{1}, n_{2}, n_{3}\right)+\sum_{n_{1}=0}^{\infty} \sum_{n_{3}=n_{1}+1 / \mu}^{\infty} p_{\mu}\left(n_{1}, n_{1}, n_{3}\right)
$$


$p\left(n_{1}, n_{2}, n_{3}\right)$ is the joint probability of having $n_{1} \mu, n_{2} \mu$ and $n_{3} \mu$ mutations respectively on the branches $(\alpha),(\beta)$ and $((\alpha \beta) \cup(\gamma))$ of the tree in Fig. 3. The factor 2 is an exchange factor which takes into account the possibility of having $n_{1}=n(\alpha)$ or equivalently $n_{1}=n(\beta)$ if $n(\alpha) \neq n(\beta)$.

Since the three events are independent, using the exponential distributions of the coalescent times $t_{n}$, i.e. of the length of the branches of the genealogical tree, we have:

$$
p\left(n_{1}, n_{2}, n_{3}\right)=2 \int_{0}^{\infty} \int_{0}^{\infty} p_{\mu}\left(n_{1} \mid t_{3}\right) p_{\mu}\left(n_{2} \mid t_{3}\right) p_{\mu}\left(n_{3} \mid 2 t_{2}+t_{3}\right) 3 e^{-t_{2}+3 t_{3}} d t_{2} d t_{3},
$$

where the factor 2 comes from the Jacobian of the transformation $t^{\prime}=t^{\prime}\left(t_{2}, t_{3}\right)=2 t_{2}+t_{3}$.

The conditional probabilities in the integral are all of the form $p_{\mu}(n \mid t)$ as given by the expression

$$
p_{\mu}(n \mid t)=\left(\begin{array}{c}
t N \\
n \mu
\end{array}\right)\left(\frac{\mu}{2 N}\right)^{n \cdot \mu}\left(1-\frac{\mu}{2 N}\right)^{t N-n \cdot \mu} \sim \frac{e^{-\mu t / 2}(\mu t / 2)^{n \mu}}{(n \mu) !},
$$

which is easily derivable from equation (8) and the second approximations holds for large populations $(N \rightarrow \infty)$.

In the same way one has that the probability $P(B)$ of event $B$ is the probability of having $n_{1}>n_{2}$ and $n_{3}=n_{1}$. Then we obtain:

$$
P(B)=2 \sum_{n_{1}=1 / \mu}^{\infty} \sum_{n_{2}=0}^{n_{1}-1 / \mu} p_{\mu}\left(n_{1}, n_{2}, n_{1}\right)
$$

where the factor 2 is also an exchange factor.

Finally, for the probability $P(C)$ of event $C$, that is the probability of having $n_{1}=n_{2}=n_{3}$, we can write:

$$
P(C)=\sum_{n_{1}=0}^{\infty} p_{\mu}\left(n_{1}, n_{1}, n_{1}\right)
$$

where, of course, there is no exchange factor.

Putting together all the different terms, the resulting expression is:

$$
P(R)=3 \int_{0}^{\infty} \int_{0}^{\infty} P\left(R \mid t_{2}, t_{3}\right) e^{-\left(3 t_{3}+t_{2}\right)} d t_{2} d t_{3}
$$

and

$$
P\left(R \mid t_{2}, t_{3}\right)=\sum_{n_{1}=\frac{1}{\mu}}^{\infty} \sum_{n_{2}=0}^{n_{1}-\frac{1}{\mu}} \sum_{n_{3}=n_{1}+\frac{1}{\mu}}^{\infty} 2 p_{\mu}\left(n_{1} \mid t_{3}\right) p_{\mu}\left(n_{2} \mid t_{3}\right) p_{\mu}\left(n_{3} \mid t_{3}+2 t_{2}\right)+
$$




$$
\begin{aligned}
& +\sum_{n_{1}=0}^{\infty} \sum_{n_{3}=n_{1}+\frac{1}{\mu}}^{\infty} p_{\mu}\left(n_{1} \mid t_{3}\right)^{2} p_{\mu}\left(n_{3} \mid t_{3}+2 t_{2}\right)+ \\
& +\sum_{n_{1}=\frac{1}{\mu}}^{\infty} \sum_{n_{2}=0}^{n_{1}-\frac{1}{\mu}} p_{\mu}\left(n_{1} \mid t_{3}\right) p_{\mu}\left(n_{2} \mid t_{3}\right) p_{\mu}\left(n_{1} \mid t_{3}+2 t_{2}\right)+ \\
& +\sum_{n_{1}=0}^{\infty} \frac{1}{2} p_{\mu}\left(n_{1} \mid t_{3}\right)^{2} p_{\mu}\left(n_{1} \mid t_{3}+2 t_{2}\right)
\end{aligned}
$$

Finally, the probability of a wrong reconstruction is $P(W)=1-P(R)$.

[1] A. Dalal and E. Schmutz, Compositions of Random Functions on a Finite Set, Electronic Journal of Combinatorics, 9, R26, (2002).

[2] B. Derrida and D. Bessis, Statistical properties of valleys in the annealed random map model, Jourmal of Physics A: Mathematical and General, 21, (1999), L509-L515.

[3] B. Derrida and B. Jung-Muller, The genealogical tree of a chromosome, Journal of Statistical Physics, 94, (1999), 277-298.

[4] B. Derrida and L. Peliti, Evolution in a flat fitness landscape, Bulletin of Mathematical Biology 53, (1991), 355-382.

[5] R. D. Gray and Q. D. Atkinson, Language-tree divergence times support the Anatolian theory of Indo-European origin. Nature 426, (2003), 435-439

[6] R. D. Gray and F. M, Jordan, Language trees support the express-train sequence of Austronesian expansion. Nature 405, (2000), 1052-1055

[7] J. F. C. Kingman, The Coalescent, Stochastic Processes and their Applications, 13, (1982), 235-248.

[8] J. F. C. Kingman, Origins of the Coalescent: 1974-1982, Genetics, 156, (December 2000), 1461-1463.

[9] M. Krings, C. Capelli, F. Tschentscher, H. Geisert, S. Meyer, A. von Haeseler, K. Grossshmidt, G. Possnert, M. Paunovic and S. Pääbo, A view of Neanderthal genetic diversity, Nature Genetics, 26, (2000), 144-146.

[10] M. Krings, A. Stone, R. W. Schmitz, H. Krainitzki, M. Stoneking and S. Pääbo, Neanderthal DNA sequences and the origin of modern humans, Cell, 90, (1997), 19-30.

[11] D. F. Robinson and L. R. Foulds, Comparison of phylogenetic trees. Math. Biosci. 53, (1/2) 
(1981)

[12] F. Petroni, L. Prignano and M. Serva, Family trees: languages end genetics, Markov processes and related fields, RANDOM INHOMOGENEOUS SYSTEMS Jenuary 22-23 2008 (in publication)

[13] F. Petroni and M. Serva, Languages distances and trees reconstruction. Journal of Statistical Mechanics: theory and experiment, (2008), P08012.

[14] M. Serva and F. Petroni, Indo-European languages tree by Levenshtein distance. EuroPhysics Letters 81, (2008), 68005

[15] M. Serva, Lack of self averaging in family trees. Physica A 332, (2004), 387-393

[16] M. Serva, On the genealogy of populations: trees, branches and offspring. Journal of Statistical Mechanics: theory and experiment, (2005), P07011.

[17] P.H.A. Sneath and R.R. Sokal, Numerical Taxonomy. Freeman, San Francisco (1973).

[18] S. Tavare, Line-of-descent and genealogical processes and their applications in population genetics models, Theoretical Population Biology, 26, (1984), 119-164. 